\title{
Intravascular large B-cell lymphoma involving hemangiomas: an unusual presentation of a rare neoplasm
}

\author{
Brigitte K Nixon ${ }^{1}$, Steven J Kussick ${ }^{2}$, Michael J Carlon ${ }^{3}$ and Brian P Rubin ${ }^{1}$ \\ ${ }^{1}$ Department of Anatomic Pathology, University of Washington Medical Center, Seattle, WA, USA; \\ ${ }^{2}$ Department of Laboratory Medicine, University of Washington Medical Center, Seattle, WA, USA and ${ }^{3}$ Group \\ Health Cooperative Hospital, Seattle, WA, USA
}

\begin{abstract}
We report the clinicopathological features of two cases of intravascular large B-cell lymphoma involving cutaneous hemangiomas. The cases were identified from the consultation files of two of the authors. Both patients were women, 64 and 55 years of age, who presented with long-standing cutaneous hemangiomas of the posterior scalp and left shoulder, respectively. The lesions were brought to medical attention by an increase in size and change in color. Biopsies and immunohistochemical evaluation of the hemangiomas revealed extensive involvement by intravascular large B-cell lymphoma. The neoplastic cells were diffusely positive for CD20 in both cases and negative for CD3, pan-cytokeratin (AE1/AE3), epithelial membrane antigen, S-100, Factor VIII-related antigen, CD34 and CD31. Disease was limited to the hemangiomas in both patients. Treatment consisted of chemotherapy (both patients) and adjuvant radiation therapy (one patient). One patient had a recurrence of disease 33 months after initial diagnosis, leading to an autologous stem cell transplant. The other patient is without evidence of disease $\mathbf{2 7}$ months after initial diagnosis. Although this is a rare neoplasm, it is important to consider intravascular large B-cell lymphoma in the differential diagnosis of vascular lesions containing intravascular neoplastic cells.
\end{abstract}

Modern Pathology (2005) 18, 1121-1126. doi:10.1038/modpathol.3800397; published online 1 April 2005

Keywords: intravascular lymphoma; large B-cell lymphoma; hemangioma

Intravascular large B-cell lymphoma, also referred to as intravascular lymphomatosis, malignant angioendotheliosis, and angiotropic large-cell lymphoma, is a rare type of non-Hodgkin's lymphoma with an unusually aggressive clinical course. It was originally described in 1959 as an endothelial-derived neoplasm with cutaneous involvement. ${ }^{1,2}$ However, it is now understood to be a B-cell lymphoma based on immunohistochemical and molecular techniques, ${ }^{3-5}$ and is recognized as a distinct subtype of diffuse large B-cell lymphoma under the World Health Organization (WHO) classification of hematolymphoid neoplasms. ${ }^{6}$ Although a case of T-cell intravascular lymphoma has been reported in the literature, T-cell intravascular lymphoma is not a recognized entity under the WHO classification system. ${ }^{7}$ Intravascular lymphoma most commonly

Correspondence: Dr BP Rubin, MD, PhD, Department of Anatomic Pathology, University of Washington Medical Center, 1959 NE Pacific Street, Box 356100, Seattle, WA 98195, USA.

E-mail: bprubin@u.washington.edu

Received 14 October 2004; revised and accepted 20 January 2005; published online 1 April 2005 occurs in the central nervous system and skin, but it can involve any organ/site including lymph nodes, spleen, kidney, lung, endocrine organs, and heart. ${ }^{4,8-18}$ A case of intravascular large B-cell lymphoma involving a pre-existing cutaneous hemangioma has been described in the English language literature. ${ }^{19}$ We present the clinicopathologic features of two additional cases of intravascular large B-cell lymphoma involving pre-existing cutaneous hemangiomas.

\section{Materials and methods}

Two cases of intravascular large B-cell lymphoma involving cutaneous hemangiomas were identified from the consultation files of two of the authors (BPR and SJK).

Sections of each case were stained with hematoxylin and eosin (H\&E). Immunohistochemistry was performed on the primary lesions of both cases using the streptavidin-biotin-peroxidase detection technique (ABC detection system, Vector Laboratories, Burlingame, CA, USA) and commercially 
available antibodies to the following antigens: CD3 (PS-1; 1:100; Vector Laboratories), CD5 (4C7; 1:50; Vector Laboratories), CD20 (L-26; 1:400; DAKO, Carpinteria, CA, USA), pan-cytokeratin (AE1/AE3; 1:500; Boehringer/Mannheim, Mannheim, Germany), epithelial membrane antigen (EMA) (E29; 1:1000; DAKO), S-100 (polyclonal; 1:8000; DAKO), Factor VIII-related antigen (FVIII-RAg) (F8168; 1:50; DAKO), CD34 (Q bend10; 1:100; DAKO), and CD31 (JC/70A; 1:250; DAKO). Immunohistochemistry was performed on the recurrent lesion (case 2) using the streptavidin-biotin-peroxidase detection technique and commercially available antibodies to the following antigens: BCL-6 (P1F6; 1:25; Novocastra, United Kingdom), cyclin D1 (P2D11F11; 1:20; Novocastra), human herpesvirus-8 (HHV8) (polyclonal; 1:1000; Advanced Biotechnologies, Columbia, MD, USA), and MUM-1 (MUMIP; 1:1000; DAKO). Positive and negative controls were used for each immunohistochemical stain.

In situ hybridization with a biotinylated complementary DNA (cDNA) probe to EBER1 messenger RNA (mRNA) of Epstein-Barr virus was also performed on the case 2 recurrence. Localization was via a three-step procedure employing a mouse anti-biotin antibody, followed by a biotinylated anti-mouse antibody, and finally an avidin-biotin complex using nickel chloride enhanced 3,3'-diaminobenzidine as the chromogen. Positive and negative controls were used.

Four-color flow cytometry was performed on a thigh (skin) nodule from the recurrence of case 2, using a Coulter Epics XL flow cytometer and commercially available antibodies to the following antigens: CD45 (J.33; Beckman Coulter, Hialeah, FL, USA), CD5 (BL1a; Beckman Coulter), CD19 (HD327; Beckman Coulter), lambda (1-155-2; Becton Dickinson (BD) Biosciences, San Jose, CA, USA), kappa (TB 28-2; BD Biosciences), CD10 (HI10a; BD Biosciences) and CD20 (L27; BD Biosciences).

PCR evaluation for B-cell clonality was attempted on paraffin-embedded tissue from both the original diagnostic specimen, and the recurrence, of case 2 . Insufficient tissue remained from the case 1 block to enable PCR evaluation. DNA was extracted from deparaffinized sections using the Puregene kit (Gentra Systems, Minneapolis, MN, USA) according to the method of the manufacturer, precipitated with $80 \%$ ethanol, and resuspended in Tris-buffered EDTA. For the PCR analysis of immunoglobulin heavy chain (IgH) gene rearrangement, 500 ng of DNA was incubated with $1 \mathrm{U}$ of Taq polymerase (Amplitaq, Applied Biosystems, Foster City, CA, USA) and $200 \mu \mathrm{M}$ dNTPs, in buffer containing $12 \mathrm{mM}$ Tris (pH 8.3), $1.2 \mathrm{mM} \mathrm{MgCl}_{2}, 60 \mathrm{mM} \mathrm{KCl}$, and $0.6 \mu \mathrm{g} / \mu \mathrm{l}$ bovine serum albumin. Consensus primers against the CDRIII (5'-ACACGGC(C/T) GT(G/A)TATTACTGT-3') and $\mathrm{J}_{\mathrm{H}}$ (5'-ACCTGAGGA GACGGTGACC-3') regions of the IgH gene were used for the PCR amplifications. The primer sets included a fluorescent (Hex)-labeled primer, allowing the products to be size-fractionated by capillary electrophoresis using an Applied Biosystems ABI 310 Sequence Analyzer with Genescan software. Positive and negative controls for clonality were used in all assays.

\section{Results/case histories}

\section{Case 1}

A 64-year-old woman presented with a posterior scalp lesion of 1-year duration that was brought to medical attention by local irritation and discomfort. She denied fever and weight loss. Biopsy of the lesion revealed a capillary hemangioma with extensive involvement by intravascular neoplastic cells (Figure 1a and b). The patient had no other skin lesions to suggest widespread cutaneous involvement. Computed tomography (CT) scan of the head/neck, chest, abdomen, and pelvis showed no other evidence of disease. Evaluation of bone marrow biopsy/aspirate and cerebrospinal fluid (CSF) showed no evidence of involvement by the neoplastic process. She received three cycles of chemotherapy (CHOP; cyclophosphamide, doxorubicin, vincristine, and prednisone) and adjuvant external beam radiation therapy to the scalp (3750 cGy in $250 \mathrm{cGy}$ fractions using $9 \mathrm{MeV}$ electrons). The patient tolerated the treatment well, with only local alopecia in the field of radiation. She has been in clinical remission for 27 months.

\section{Case 2}

A 55-year-old woman presented with a long-standing, bluish-red lesion on her left shoulder that was brought to medical attention due to a recent increase in size. She had no other complaints. Biopsy of the lesion revealed a capillary hemangioma with extensive involvement by intravascular neoplastic cells (Figure 1c). She had no other skin lesions to suggest widespread cutaneous involvement. Bilateral bone marrow biopsies revealed no evidence of neoplasm. CT scans of the chest, abdomen, and pelvis revealed no evidence of disseminated disease. A whole-body gallium scan was also negative for disseminated disease. The patient received six cycles of chemotherapy (CHOP). She remained in clinical remission until 33 months after the initial diagnosis. At that time, she presented with a $2 \mathrm{~cm}$ subcutaneous thigh nodule and inguinal lymphadenopathy. Flow cytometry was performed on the thigh (skin) nodule and was consistent with a recurrence (Figure 3). Histologic evaluation of the thigh (skin) nodule confirmed recurrent large B-cell lymphoma. The patient ultimately underwent autologous stem cell transplantation for her recurrent lymphoma, and has remained in clinical remission in the 15 months since the transplantation. 

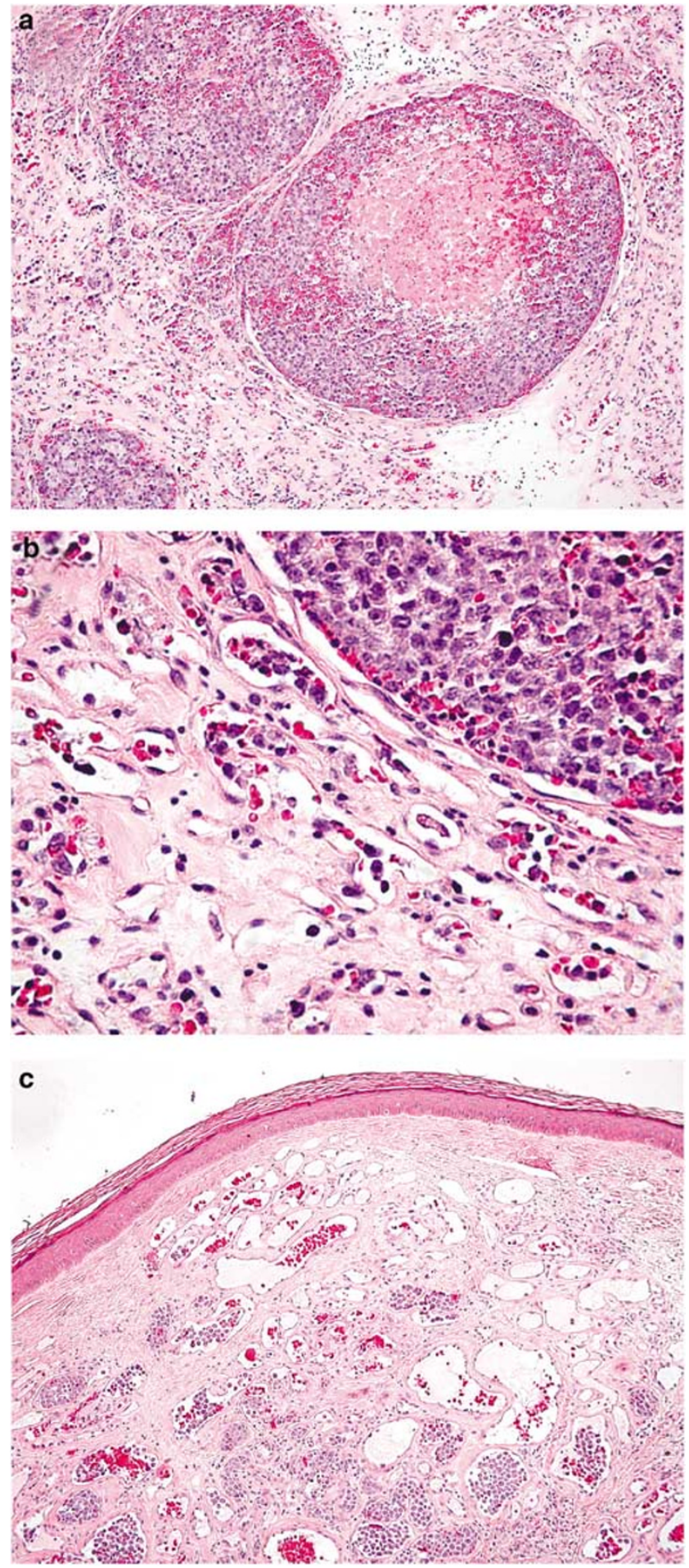

Figure 1 Histological features of intravascular lymphoma. (a) Low-power hematoxylin and eosin (H\&E)-stained section of Case 1 shows prominent distention of vascular structures by neoplastic cells with central comedo-type necrosis. (b) Highpower H\&E-stained section of Case 1 shows the large, vesicular nuclei and prominent nucleoli of the neoplastic cells. (c) Lowpower H\&E-stained section of Case 2 reveals a dermal hemangioma with extensive intravascular involvement by neoplastic cells.

\section{Histologic features}

The histologic features of both cases were similar at initial presentation. Both lesions consisted of a proliferation of dilated vascular channels lined by endothelial cells, consistent with capillary hemangiomas (Figure 1). Many of the vascular channels were distended by groups of frankly neoplastic cells with large, vesicular nuclei and prominent nucleoli. Mitotic figures (including atypical mitotic figures) numbered up to 11 per 10 high-power fields in the two cases. Central, comedo-type necrosis was identified in case 1. These morphological features suggested a differential diagnosis of metastatic carcinoma, malignant melanoma, epithelioid angiosarcoma, and intravascular large B-cell lymphoma. The biopsy of the thigh (skin) nodule from case 2 at the time of recurrence revealed a lymph node with complete effacement of the architecture and replacement of the lymph node by large, neoplastic cells having vesicular nuclei, and prominent nucleoli. Although these neoplastic cells had similar cytologic features to those in the primary lesion, mitotic figures were much more numerous in the recurrence, numbering over 50 per 10 highpower fields. Groups of neoplastic cells were also located in the vascular spaces adjacent to the lymph node, indicating an intravascular component in the recurrence.

\section{Ancillary studies}

The neoplastic cells in the primary lesions of both cases were diffusely positive for CD20 (Figure 2a) and negative for CD3, pan-cytokeratin (AE1/AE3), EMA, S-100, FVIIIRAg, CD34, and CD31. CD34 immunohistochemistry highlighted the intravascular location of the neoplastic B cells (Figure 2b). The neoplastic cells in case 2 showed low to moderate staining for CD5 while case 1 was negative (data not shown). Flow cytometry of the thigh nodule at the time of recurrence of case 2 demonstrated a population of neoplastic cells having large size, dim to intermediate CD19, intermediate to bright CD20, bright CD45, dim kappa light chain restriction, and aberrant coexpression of dim to intermediate CD5 (Figure 3). By immunohistochemistry, the neoplastic cells of the thigh nodule were diffusely positive for CD5 and MUM-1, focally positive for BCL-6 (10\% of cells with immunoreactivity), and negative for cyclin D1 and HHV8 (data not shown). In addition, these cells were negative for EBV by in situ hybridization using a probe for EBER1 mRNA. Molecular evaluation of the B cells (immunoglobulin heavy chain gene PCR) in both the initial biopsy and the recurrence of case 2 was attempted in an effort to confirm clonal identity. However, these studies were noninformative, as insufficient DNA was recovered from the initial biopsy to enable PCR 
to be performed, while only the polyclonal background B cells amplified from the recurrence. Given the clear flow cytometric identification of
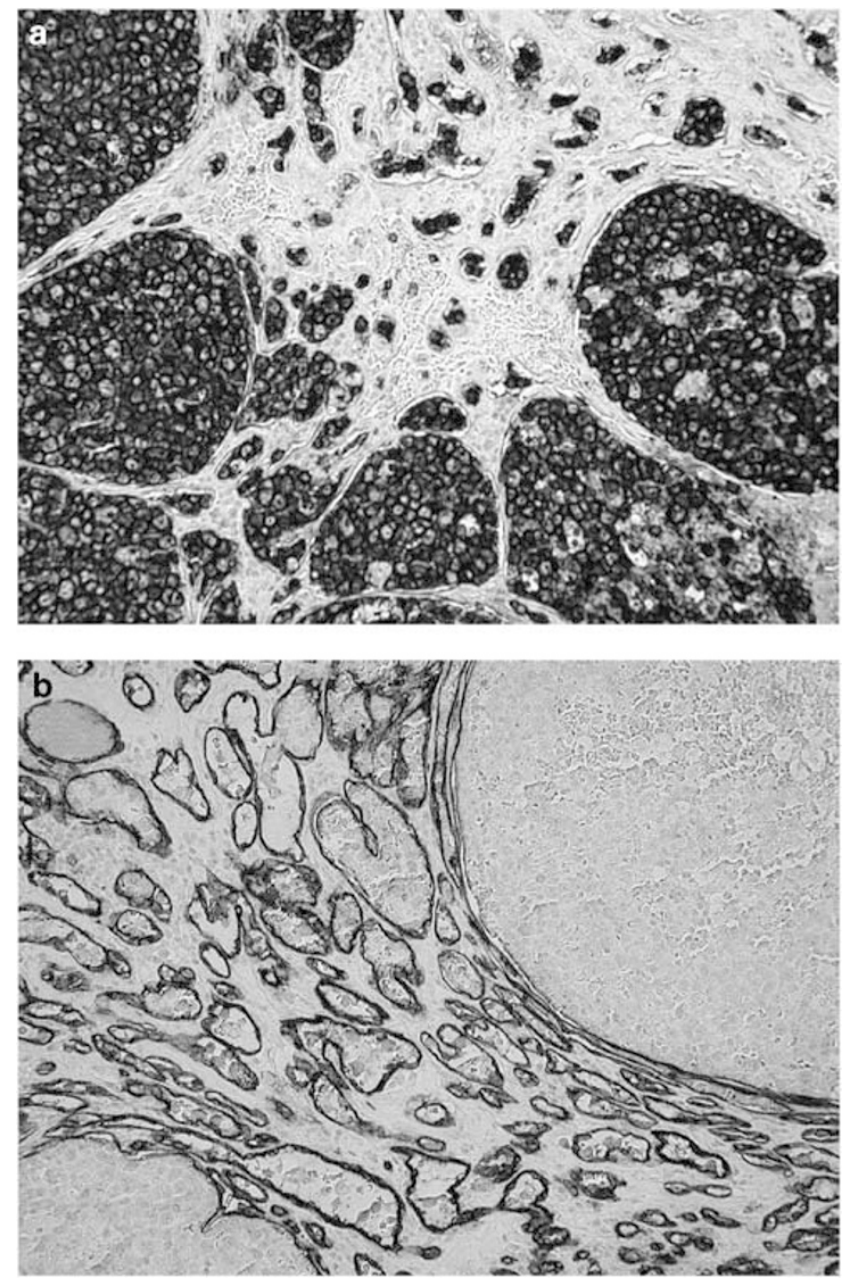

Figure 2 Immunohistochemical features of intravascular lymphoma. (a) The neoplastic cells in Case 1 are diffusely and strongly positive for CD20. (b) Immunohistochemical staining for CD34 highlights the complex network of blood vessels within the hemangioma. The neoplastic cells within the distended blood vessels are negative for CD34. clonality (kappa light chain-restriction) in the recurrence, the polyclonal PCR represents a falsenegative, presumably due to the phenomenon of somatic hypermutation.

\section{Discussion}

The morphologic and immunohistochemical features of these lesions are diagnostic of intravascular large B-cell lymphoma involving pre-existing dermal-based hemangiomas. Although this presentation is rare for intravascular large B cell lymphoma, one case has been reported previously in the English language literature. ${ }^{19}$ In that report, the patient, who initially received close observation followed by chemotherapy at 10 months after diagnosis, died with disseminated disease 23 months after initial presentation. In our study, the patient who received 3 cycles of chemotherapy (CHOP) and adjuvant localized external beam radiation remains in clinical remission 27 months after initial diagnosis. The second patient in our study, who received 6 cycles of chemotherapy (CHOP) without adjuvant radiation, had a recurrence of disease 33 months after initial diagnosis, necessitating autologous stem cell transplantation for the patient to achieve a clinical remission.

The prognosis of patients with intravascular large $\mathrm{B}$-cell lymphoma is generally poor. In a review of 86 cases of intravascular large B-cell lymphoma, there was a median survival of 5 months from the date of clinical presentation. ${ }^{20}$ Prolongation of survival has been reported in patients with intravascular large B-cell lymphoma who are treated with chemotherapy. ${ }^{21}$ In a review of 35 patients who received chemotherapy, $54 \%$ had a complete remission. ${ }^{21}$ Median survival for those who did not respond to chemotherapy, or only partially responded, was 10.5 months. $^{21}$

Although the number of cases in our study is too small to develop broad conclusions about the clinical behavior of intravascular large B-cell lymphoma involving cutaneous hemangiomas, the relatively favorable outcome in these two cases
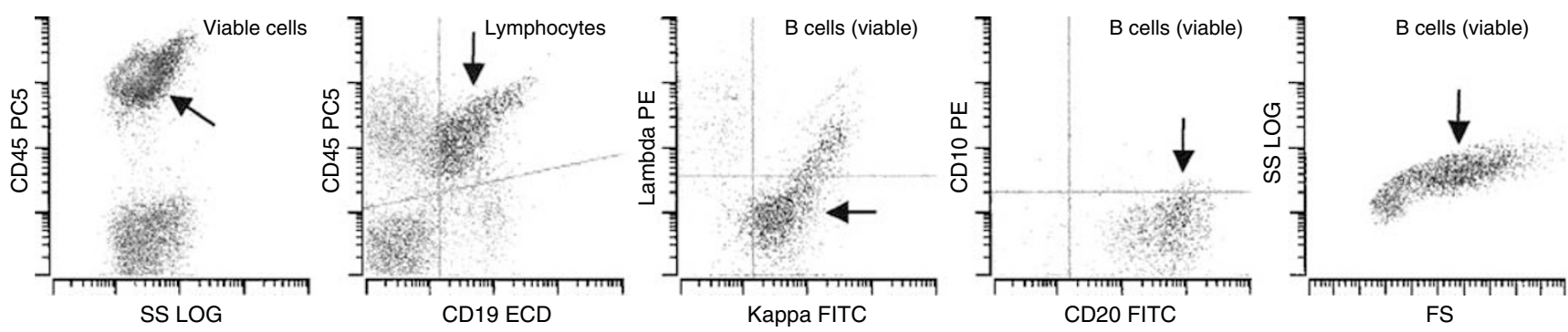

Figure 3 Four-color flow cytometric analysis of the thigh (skin) biopsy from Case 2 at the time of recurrence reveals an abnormal B-cell population having large cell size (increased forward light scatter, or FS, on the right-most dot-plot), dim kappa light chain restriction, dim to intermediate CD19, intermediate to bright CD20, bright CD45, and aberrant coexpression of dim to intermediate CD5. In each dot-plot, the abnormal B-cell population is denoted by the arrow. 
may have resulted from cutaneous signs and symptoms leading to relatively early disease identification and treatment. It is possible that the recurrence in case 2 was related to the lack of adjuvant radiation therapy, since patient 1 actually received three fewer cycles of chemotherapy (CHOP). Alternatively, it is possible that the adverse prognostic features of the neoplastic cells in case 2 were related to the recurrence. These adverse prognostic features included the nongerminal center B-cell phenotype (expression of MUM-1 without CD10), ${ }^{22}$ as well as CD5 coexpression. ${ }^{23}$ Despite the CD5-coexpression, the recurrent lesion in case 2 did not express cyclin D1, excluding the possibility of a pleomorphic variant of mantle cell lymphoma. The neoplastic cells from case 1 were CD5-negative, but insufficient tissue remained in the block to assess germinal center-associated antigen expression.

The mechanism responsible for the predilection of intravascular large B-cell lymphoma for blood vessels is not known. Some researchers have shown that the neoplastic lymphocytes of intravascular large B-cell lymphoma lack specific surface adhesion molecules, like CD29 and CD54, that are necessary for transvascular migration. ${ }^{24}$ However, it is not clear what proportion of intravascular large B-cell lymphomas lack these adhesions molecules, nor whether additional mechanisms may be responsible for the intravascular location of these neoplasms. In addition, although other cases of CD5-positive intravascular large B-cell lymphoma have been reported in the literature ${ }^{25-27}$ it remains unclear whether there is any mechanistic relationship between CD5 coexpression and intravascular localization in these lymphomas. Although one study of intravascular lymphoma identified bone marrow involvement in four of five cases, and peripheral blood involvement in all five cases, ${ }^{25}$ we have not been able to detect the neoplastic cells in the peripheral blood or bone marrow by flow cytometry in case 2, even though this patient has been extensively followed by our institution because of the recurrent lesion. The staging bone marrow in case 1 was reportedly negative by morphology; however, to our knowledge, neither that patient's blood nor bone marrow was ever evaluated by flow cytometry.

In summary, we report two cases of intravascular large B-cell lymphoma that were identified in association with pre-existing cutaneous hemangiomas. Intravascular large B-cell lymphoma should be included in the differential diagnosis of vascular lesions containing intravascular, malignant-appearing cells. The relatively favorable outcome of the patients in this study, compared to the outcomes of other patients with intravascular large B-cell lymphoma, may be related to earlier detection and treatment due to the cutaneous location of these lesions.

\section{References}

1 Pfleger L, Tappeiner J. Zur Kenntnis der systemisierten endotheliomatose der cutanen blutgefabe. Hautarzt 1959;10:359-363.

2 Tappeiner J, Pfleger L. Angioendotheliomatosis proliferans systemisata: ein klinisch und histopathologisch neues Krankheitsbild. Hautarzt 1963;14:67-70.

3 Otrakji CL, Voigt W, Amador A, et al. Malignant angioendotheliomatosis-a true lymphoma: a case of intravascular malignant lymphomatosis studied by Southern blot hybridization analysis. Hum Pathol 1988;19:475-478.

4 Sheibani K, Battifora H, Winberg CD, et al. Further evidence that 'malignant angioendotheliomatosis' is an angiotropic large-cell lymphoma. N Engl J Med 1986; 314:943-948.

5 Sleater JP, Segal GH, Scott MD, et al. Intravascular (angiotropic) large cell lymphoma: determination of monoclonality by polymerase chain reaction on paraffin-embedded tissues. Mod Pathol 1994;7:593-598.

6 Warnke RA, Gatter KC. Intravascular large B-cell lymphoma. In: Jaffe ES, Harris NL, Stein H, Vardiman JW (eds). Pathology and Genetics of Tumours of the Haematopoietic and Lymphoid Tissues. IARC Press: Lyon, 2001, pp 177-178.

7 Sepp N, Schuler G, Romani N, et al. 'Intravascular lymphomatosis' angioendotheliomatosis): evidence for a T-cell origin in two cases. Hum Pathol 1990;21: 1051-1058.

8 Carroll Jr TJ, Schelper RL, Goeken JA, et al. Neoplastic angioendotheliomatosis: immunopathologic and morphologic evidence for intravascular malignant lymphomatosis. Am J Clin Pathol 1986;85:169-175.

9 Cheng FY, Tsui WM, Yeung WT, et al. Intravascular lymphomatosis: a case presenting with encephalomyelitis and reactive haemophagocytic syndrome diagnosed by renal biopsy. Histopathology 1997;31: $552-554$

10 Fulling KH, Gersell DJ. Neoplastic angioendotheliomatosis. Histologic, immunohistochemical, and ultrastructural findings in two cases. Cancer 1983;51: 1107-1118.

11 Kitagawa M, Matsubara O, Song SY, et al. Neoplastic angioendotheliosis. Immunohistochemical and electron microscopic findings in three cases. Cancer 1985;56:1134-1143.

12 Mori S, Itoyama S, Mohri N, et al. Cellular characteristics of neoplastic angioendotheliosis. An immunohistological marker study of 6 cases. Virchows Arch A Pathol Anat Histopathol 1985;407:167-175.

13 Petito CK, Gottlieb GJ, Dougherty JH, et al. Neoplastic angioendotheliosis: ultrastructural study and review of the literature. Ann Neurol 1978;3:393-399.

14 Scott PW, Silvers DN, Helwig EB. Proliferating angioendotheliomatosis. Arch Pathol 1975;99:323-326.

15 Wang BY, Strauchen JA, Rabinowitz D, et al. Renal cell carcinoma with intravascular lymphomatosis: a case report of unusual collision tumors with review of the literature. Arch Pathol Lab Med 2001;125: 1239-1241.

16 Wick MR, Banks PM, McDonald TJ. Angioendotheliomatosis of the nose with fatal systemic dissemination. Cancer 1981;48:2510-2517.

17 Wick MR, Mills SE. Intravascular lymphomatosis: clinicopathologic features and differential diagnosis. Semin Diagn Pathol 1991;8:91-101. 
18 Wrotnowski U, Mills SE, Cooper PH. Malignant angioendotheliomatosis. An angiotropic lymphoma? Am J Clin Pathol 1985;83:244-248.

19 Rubin MA, Cossman J, Freter CE, et al. Intravascular large cell lymphoma coexisting within hemangiomas of the skin. Am J Surg Pathol 1997;21:860-864.

20 Domizio P, Hall PA, Cotter F, et al. Angiotropic large cell lymphoma (ALCL): amorphological, immunohistochemical and genotypic studies with analysis of previous reports. Hematol Oncol 1989;7:195-206.

21 DiGiuseppe JA, Nelson WG, Seifter EJ, et al. Intravascular lymphomatosis: a clinicopathologic study of 10 cases and assessment of response to chemotherapy. J Clin Oncol 1994;12:2573-2579.

22 Hans CP, Weisenburger DD, Greiner TC, et al. Confirmation of the molecular classification of diffuse large B-cell lymphoma by immunohistochemistry using tissue microarray. Blood 2004;103:275-282.
23 Yamaguchi $\mathrm{M}$, Seto $\mathrm{M}$, Okamoto $\mathrm{M}$, et al. De novo CD5+ diffuse large B-cell lymphoma: a clinicopathologic study of 109 patients. Blood 2002;99:815-821.

24 Ponzoni M, Arrigoni G, Gould VE, et al. Lack of CD 29 ( $\beta 1$ integrin) and CD 54 (ICAM-1) adhesion molecules in intravascular lymphomatosis. Hum Pathol 2000;31: 220-226.

25 Estalilla OC, Koo CH, Brynes RK, et al. Intravascular large B-cell lymphoma. A report of five cases initially diagnosed by bone marrow biopsy. Am J Clin Pathol 1999;112:248-255.

26 Khalidi HS, Brynes RK, Browne P, et al. Intravascular large B-cell lymphoma: the CD5 antigen is expressed by a subset of cases. Mod Pathol 1998;11:983-988.

27 Ferry JA, Harris NL, Picker LJ, et al. Intravascular lymphomatosis (malignant angioendotheliomatosis). A B-cell neoplasm expressing surface hoing receptors. Mod Pathol 1988;1:444-451. 